\title{
Effect of Angular Velocity on Mass Fraction Distribution for Jets Impinging on Airfoil Leading-Edge Cavity
}

\author{
Amin Safi ${ }^{1}$, Mohammad O. Hamdan ${ }^{2}$, Emad Elnajjar ${ }^{1}$ \\ ${ }^{1}$ United Arab Emirates University \\ AlAin, Abu Dhabi, UAE \\ 201590089@uaeu.ac.ae; eelnajjar@uaeu.ac.ae \\ ${ }^{2}$ American University of Sharjah \\ University City, Sharjah, UAE \\ mhamdan@aus.edu
}

\begin{abstract}
In the present study, the effect of angular velocity on mass fraction per jet is evaluated using three dimensional numerical model. A steady state model with constant wall temperature is used to investigate the performance of seven jets impinging an airfoil leading edge cavity. The numerical simulation is conducted using ANSYS - fluent release 19.1. The study analyse the impact of jet location, jet Reynolds number and rotation number on flow behaviour and how they impact cross flow interaction. The results show that the amount of mass fraction depends mainly on channel size, jet size, jet location, rotation number and Reynolds number. The highest mass fraction is found for jets near the flow outlet while the lowest mass fraction is found from the jets in farthest distance from the outlet. Under angular velocity, that mass fraction increases with rotation number for the jet near the outlet due to centrifugal force, while decreases for the jets near the rotation axis. The rotation caused higher maximum temperature at low Reynolds number $(7,500$ and 10,000$)$ while it produced lower maximum temperature at high Reynolds number (20,000 and 30,000).
\end{abstract}

Keywords: Jet impingements; mass fraction; leading edge cavity; rotating airfoil; effect of jet location.

\section{Introduction}

Gas turbine engines are widely used for aircraft propulsion, land based power generation, marine applications, tanks and industrial applications [1]. Increasing the efficiency of the cooling system plays vital role to develop gas turbine engines. The most critical region in the turbine blade with highest thermal loads is the blade leading edge. The leading edge of the blade faces the maximum thermal load compare to the rest of the gas turbine components since it is the first region after the combustion chamber [2]. One of the main factor to increase the power and efficiency of gas turbine engines is increasing the temperature at the inlet of turbine [2]. The high temperature at the leading edge reduces the life expectancy of the blade. Having the blade at $30{ }^{\circ} \mathrm{C}$ higher than designed temperature can reduce the blade life time by 50\% [3]. Jet impingement is an effective way to provide localized cooling [4-8]. Film cooling is another technique used to shield the blade surface by injecting relatively cooler air over the blade to form a blanket of air to insulate the blade from external extreme conditions $[9,10]$.

The proximity of the leading edge to the thickest section in the airfoil allows the implementation of internal jet impingement cooling technique. Due to the higher performance and ability to enhance the local heat transfer coefficient, this method has a high impact on life time of the blade compared to the other techniques [11]. Florschuetz [12] has shown that cross flow has a negative effect on heat transfer to the target impingement surface. For the curve surfaces, Chupp et al. [12] has shown that by increasing the curvature of the target surface, the average Nusselt number ratio also increases. More uniform Nusselt number distribution occurs in sharper nose compared to the smoother blunt nose as reported by Bunker and Metzger [13]. Due to complexity, many of these papers did not consider one of the major factor on heat transfer coefficient which is rotation effect. Epstein et al. [14] reported that rotation cuts around 30\% of impingement heat transfer below the coefficients in a nonrotating blades. In 1996, effect of rotation direction on the angle of jet impingement direction has been investigated by Mattern and Hennecke [15]. They used naphthalene sublimation technique in their experiment and showed that the maximum effect of the jet direction is when it is perpendicular to the rotation where the heat transfer coefficient drops by $40 \%$ without considering Coriolis force [8]. 
Deng et al. [16] experimentally studied the impingement heat transfer with film extraction flow on the leading edge of rotating blades and they reported that rotation has a great effect on heat transfer and that Coriolis force is a very important factor in decreasing the heat transfer. Hong et al. [17] has investigated the effect of jet direction on heat and mass transfer of a rotating impingement jet. They reported that jet orientation has a significant effect on heat transfer coefficient and the rotating condition can negatively effect on heat transfer coefficient by 35\%. Akella and Han [18] worked on heat transfer of jet array in rotating two pass channels and reported that Coriolis and centrifugal negatively affect the heat transfer distribution in impingement region and other sides of the blade.

\section{Problem Formulation}

A schematic of the computational domain is shown in Figure 1, which resembles seven jets impinging inside semicircular channel. The dimensions of the computational domain are shown in Table 1. This study employs a threedimensional numerical simulation which is conducted using ANSYS Fluent Release 19.1. Different value of inlet velocity has been used to obtain jet Reynolds number around the values of 7,500, 10,000, 20,000 and 30,000. Outlet pressure of zero gage pressure has been enforced at the outlet. All walls are set to adiabatic no slip condition except the curve wall where constant heat flux of $1000 \mathrm{~W} / \mathrm{m}^{2}$ has been enforced. Air is used as the cooling fluid and all its properties are set as homogenous and maintained constant throughout the simulation.
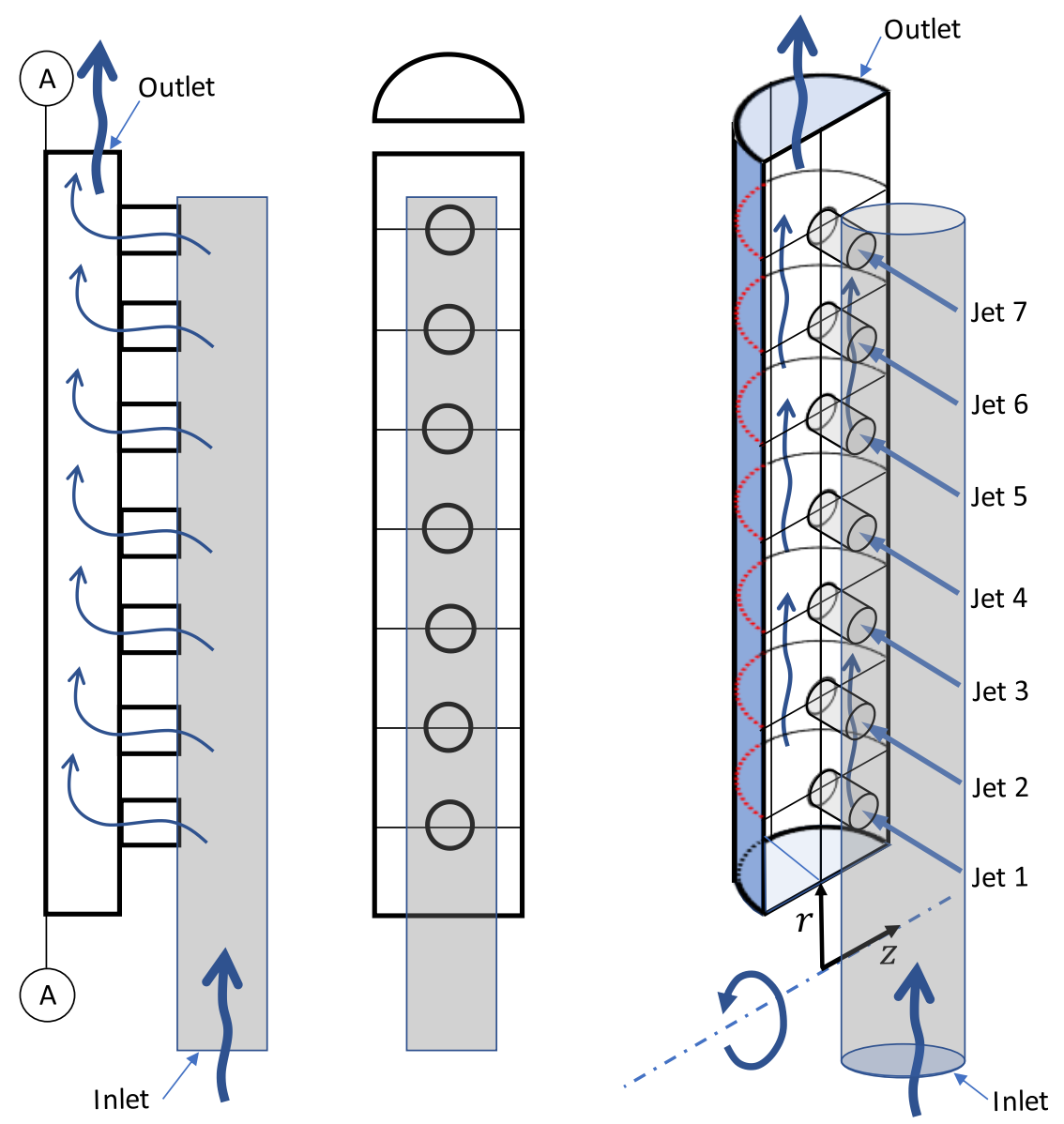

Fig. 1: Schematic of cavity being cooled using seven jets impinging on the curved surface while rotating around the z-axis. 
The ANSYS Fluent software makes use of the conservation laws for mass, momentum and energy which can be written as follow:

$$
\begin{gathered}
\frac{\partial \bar{u}_{i}}{\partial x_{i}}=0 \\
\frac{\partial \bar{u}_{i}}{\partial t}+\frac{\partial}{\partial x_{j}}\left(\bar{u}_{i} \bar{u}_{j}\right)=-\frac{1}{\rho} \frac{\partial \bar{p}}{\partial x_{i}}+v \frac{\partial^{2} \bar{u}_{i}}{\partial x_{j} \partial x_{j}}-\frac{\partial}{\partial x_{j}}\left(\overline{u_{\imath}^{\prime} u_{\jmath}^{\prime}}\right) \\
\frac{\partial T}{\partial t}+\frac{\partial}{\partial x_{i}}\left(T \bar{u}_{i}\right)=v \frac{\partial^{2} T}{\partial x_{i} \partial x_{i}}-\frac{\partial}{\partial x_{i}}\left(\overline{T^{\prime} u_{l}^{\prime}}\right)
\end{gathered}
$$

where $\rho, v, p$ and $\mu$ refer to density, velocity vector, pressure and dynamics viscosity, respectively. $T, c_{p}$ and $k$ refer to temperature, heat capacity and thermal conductivity, respectively.

Table 1: Dimension of computational domain.

\begin{tabular}{|l|c|c|}
\hline Parameters & Unit & Value \\
\hline Jet diameter $\left(d_{j}\right)$ & $\mathrm{m}$ & 0.008 \\
\hline Jet length $\left(\ell_{j}=1.5 d_{j}\right)$ & $\mathrm{m}$ & 0.012 \\
\hline Distance between jet $\left(h_{j}=4 d_{j}\right)$ & $\mathrm{m}$ & 0.032 \\
\hline Length of the semi-circular channel & $\mathrm{m}$ & 0.216 \\
\hline Diameter of the semi-circular channel & $\mathrm{m}$ & 0.05 \\
\hline
\end{tabular}

This study has adopted the SST k- $\omega$ turbulence model which is a two-equation eddy-viscosity model. The shear stress transport (SST) formulation combines the standard k- $\varepsilon$ and k- $\omega$ model through the use of blend function. The standard k- $\omega$ formulation is sued in the inner parts of the boundary layer while the standard k- $\varepsilon$ model is used in the free-stream. This allow avoiding the sensitivity of $\mathrm{k}-\omega$ model to the inlet free-stream turbulence properties.

As suggested in literature [19], the SST k- $\omega$ turbulence model is appropriate to use for rotating channel flow and flow development in a U-bend, hence it is adopted in current study with $y^{+} \sim 1$ and enhanced wall treatment and thermal effects function. More details information regarding the SST k- $\omega$ is available in literature [20].

The current problem has been solved using Semi-Implicit Method for Pressure Linked Equations (SIMPLE) algorithm. The default discretization has been adopted which implements a second-order upwind discretization for momentum and energy equations and first-order upwind discretization for Turbulence model equations. The solution convergence is set when all parameters residuals are less than $10^{-5}$ and temperature residual is less $10^{-9}$.

\section{Results and discussion}

The mass fraction represents the mass flow rate of fluid passing a jet to the total mass flow rate passing through all jets. When fluid moves in in a multi passages, it is expected that maximum flow rate will occur in the passage that has least friction losses. From the problem schematic, it is clear that one expect that jet number seven will have maximum mass fraction since all jets are of same size and the fluid is getting impinged to the same channel. The fluid leaving jet number one needs to pass over all other jet flow and is expected to travel distance to reach the exit at the top side of the channel. Hence, jet one is expected to have the least mass fraction when compared to other figures as shown 2a. As shown in figure $2 \mathrm{a}$, the angular velocity has little effect on mass fraction at high Reynolds number. Hence the flow is mainly driven by inlet pressure and flow inertia. However, as lower Reynolds number is tested as shown in figure $2 \mathrm{~b}, 2 \mathrm{c}$ and $2 \mathrm{~d}$, the effect of angular velocity become more pronounce. The increase in angular velocity causes an increase in centrifugal force which push more liquid to leave from jet 5 and 6 . This reduce the available mass flow rate at jet 1 and 2 as seen in figure $2 \mathrm{~d}$. 
The higher the angular velocity will push the fluid toward the center of the channel reduce the chances of the flow reaching the curved surface and will maximize the effect of cross flow. Also this will deteriorate jet cooling capabilities, hence higher temperature is expected as shown in figure 3. The ration of maximum temperature to inlet temperature is in figure 3. As shown in figure 3, the maximum temperature ratio decreases with the increase in Reynolds number and decrease in rotation number. The higher Reynolds number means higher jet mass flow rate and higher inertia which better heat removal from the curved surface. The higher rotation number means higher centrifugal force which promote flow, reduces the ability of jet reaching the curved surface and increase the skewness in mass fraction between jets.
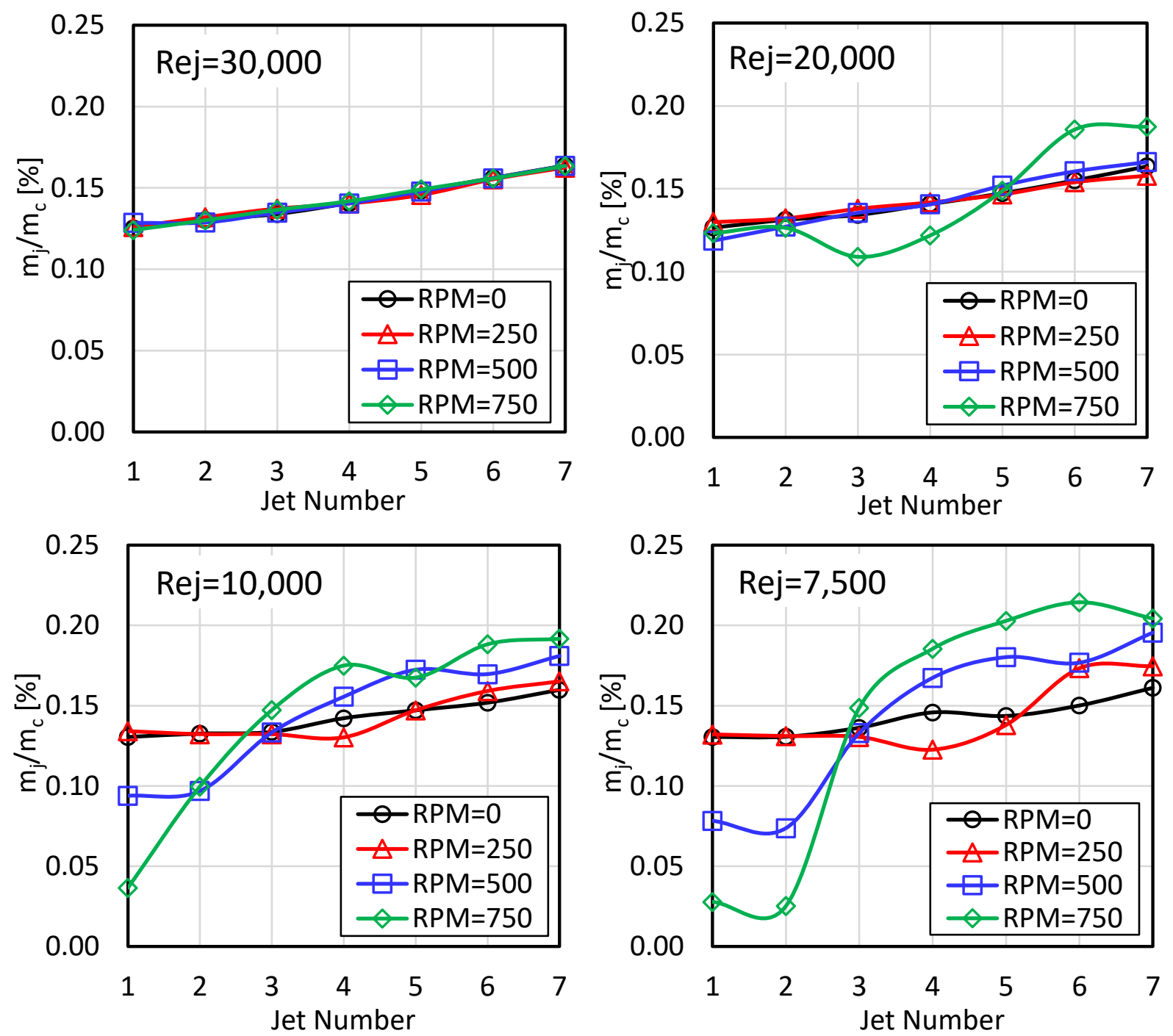

Fig. 2: Mass fraction at each jet for different Reynolds numbers (a) 30,000 (b) 20,000, (c) 10,000, and (d) 7,500. 


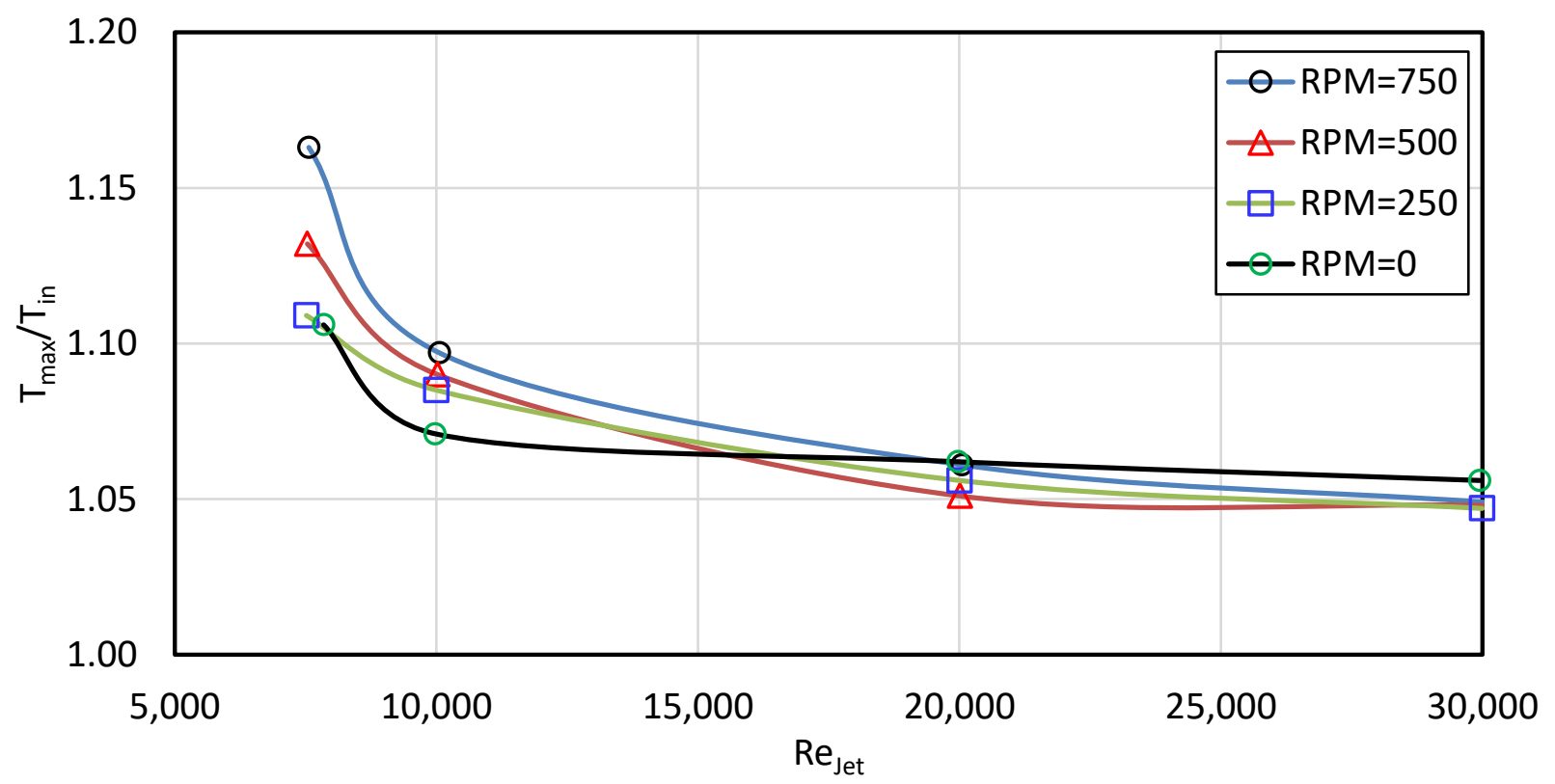

Fig. 3: Mass fraction at each jet for different Reynolds numbers (a) 30,000 (b) 20,000, (c) 10,000, and (d) 7,500.

\section{Conclusion}

More mass flow rate is injected from the jets near the exit when compared to the jets far from the channel outlet. Jet impingement is affected by channel rotation and this effect is more pronounced at low Reynolds number $(\sim 7,500)$ than at high Reynolds number $(\sim 30,000)$. In addition to cross flow effect, the variation of mass fraction due to angular velocity affects the heat transfer from heated surface. At low Reynolds number $(\sim 7,500)$, the angular velocity causes the maximum surface temperature to rise while at high Reynolds number it causes small drop in the maximum surface temperature. Gas turbines with high RPM require jet cooling using high Reynolds number jet flow.

\section{Acknowledgement}

The authors would like to extend their sincerest appreciation to United Arab Emirates University for the providing support for this research. This work was financially supported by the Research Office at UAE University under research grant contract number 31R072.

\section{References}

[1] J.-C. Han, S. Dutta, and S. Ekkad, Gas turbine heat transfer and cooling technology. CRC press, 2012.

[2] J. Taylor, "Heat Transfer Phenomena in Gas Turbines," in ASME 1980 International Gas Turbine Conference and Products Show, 1980.

[3] R. Viskanta, "Heat transfer to impinging isothermal gas and flame jets," Experimental thermal and fluid science, vol. 6, pp. 111-134, 1993.

[4] M. O. Hamdan, E. Elnajjar, and Y. Haik, "Measurement and modeling of confined jet discharged tangentially on a concave semicylindrical hot surface," Journal of Heat Transfer, vol. 133, p. 122203, 2011.

[5] M. Hamdan and M. Al-Nimr, "Thermal augmentation in internal cooling passage by converting impingement jet to induced swirl flow," in Proceedings of 6th International Conference on Computational Heat and Mass Transfer, May, 2009, pp. 18-21.

[6] E. Elnajjar, M. O. Hamdan, and Y. Haik, "Experimental investigation of internal channel cooling via jet impingement," FDMP: Fluid Dynamics \& Materials Processing, vol. 9, pp. 77-89, 2013. 
[7] E. Elnajjar, M. Hamdan, and Y. Haik, "Experimental investigation of impinging jet flow on a heated curved surface," in 6th International Conference on Thermal Engineering, 2012.

[8] M. O. Hamdan and A. Y. Hayek, "Effect of Cavity Size on Confined Slot Jet Impingement Cooling," in ASME 2013 Heat Transfer Summer Conference collocated with the ASME 2013 7th International Conference on Energy Sustainability and the ASME 2013 11th International Conference on Fuel Cell Science, Engineering and Technology, 2013.

[9] M. Al-Hemyari, M. O. Hamdan, and M. F. Orhan, "Numerical analysis of film cooling effectiveness under variable blowing ratio and injection angles," in The 3rd World Congress on Momentum, Heat and Mass Transfer (MHMT'18), Budapest, Hungary, 2018, pp. 1-8.

[10] M. Al-Hemyari, M. O. Hamdan, and M. F. Orhan, "Numerical Analysis of Film Cooling Shield Formed by Confined Jet Discharging on a Flat Plate," International Journal of Heat and Technology, vol. 37, pp. 327-333, 2019.

[11] R. Goldstein and A. Behbahani, "Impingement of a circular jet with and without cross flow," International Journal of Heat and Mass Transfer, vol. 25, pp. 1377-1382, 1982.

[12] L. Florschuetz, C. Truman, and D. Metzger, "Streamwise flow and heat transfer distributions for jet array impingement with crossflow," in ASME 1981 International Gas Turbine Conference and Products Show, 1981, pp. V003T09A005V003T09A005.

[13] R. Bunker and D. Metzger, "Local heat transfer in internally cooled turbine airfoil leading edge regions: Part Iimpingement cooling without film coolant extraction," 1990.

[14] A. Epstein, J. Kerrebrock, J. Koo, and U. Preiser, "Rotational effects on impingement cooling," in Heat Transfer and Fluid Flow in Rotating Machinery, 1987, pp. 86-102.

[15] C. Mattern and D. Hennecke, "The influence of rotation on impingement cooling," in ASME 1996 International Gas Turbine and Aeroengine Congress and Exhibition, 1996, pp. V004T09A003-V004T09A003.

[16] H. Deng, Z. Gu, J. Zhu, and Z. Tao, "Experiments on impingement heat transfer with film extraction flow on the leading edge of rotating blades," International Journal of Heat and Mass Transfer, vol. 55, pp. 5425-5435, 2012.

[17] S. K. Hong, D. H. Lee, and H. H. Cho, "Effect of jet direction on heat/mass transfer of rotating impingement jet," Applied Thermal Engineering, vol. 29, pp. 2914-2920, 2009.

[18] K. V. Akella and J.-C. Han, "Impingement cooling in rotating two-pass rectangular channels with ribbed walls," Journal of Thermophysics and heat transfer, vol. 13, pp. 364-371, 1999.

[19] T. P. Dhakal and D. K. Walters, "Curvature and rotation sensitive variants of the K-Omega SST turbulence model," in ASME 2009 Fluids Engineering Division Summer Meeting, 2009, pp. 2221-2229.

[20] F. Menter, "Zonal two equation kw turbulence models for aerodynamic flows," in 23rd fluid dynamics, plasmadynamics, and lasers conference, 1993, p. 2906. 